\title{
Patrones de consumo de alcohol, tabaco y drogas ilegales en personas transexuales
}

\section{Patterns of alcohol, tobacco, and illicit drug use among transsexuals}

\author{
Esther Gómez-Gil*, Egle Simulionyte*, Mercè Balcells-Oliveró**, Manuel Valdés*, \\ Manel Salamero*, Antonio Guillamón***, Isabel Esteva****. \\ * Clínic Institute of Neurosciences, Catalonian Gender Team, I.D.I.B.A.P.S. Hospital Clínic, Barcelona. Spain. \\ ** Clínic Institute of Neurosciences, Department of Psychiatry, Addictive Disorders Unit. Hospital Clínic, Barcelona, Spain. \\ *** Department of Psychobiology. Psychology Faculty. U.N.E.D. Madrid, Spain. **** Andalusian Gender Team, \\ Department of Endocrinology. Hospital Regional Universitario, Málaga, Spain.
}

\section{Resumen}

Este estudio evalúa los patrones de consumo de sustancias en personas transexuales de hombre a mujer (H-M) y de mujer a hombre (M-H). Un total de 251 personas transexuales (163 H-M y $88 \mathrm{M}-\mathrm{H}$ ), atendidas en la Unidad de Identidad de Género de Cataluña, completaron un cuestionario autoadministrado sobre el consumo de alcohol, tabaco, cannabis, cocaína, opiáceos y drogas de diseño. Los resultados se compararon con datos del Servicio Nacional de Salud en población general en Cataluña (estudio EDADES 2013). La prevalencia del consumo de alcohol $(70,1 \%)$, tabaco $(46,2 \%)$ y cannabis $(16,3 \%)$ actual en el total de personas transexuales de ambos sexos fue similar al de hombres en población general $(72,1 \%, 42,1 \%, 12,8 \%)$ y mayor que la prevalencia en mujeres $(57,6 \%, 35,2 \%, 5 \%)$; no se encontraron diferencias en dicho consumo entre H-M y M-H. El consumo de cocaína en H-M $(9,8 \%)$ fue casi diez veces más prevalente que en el subgrupo M-H $(1,1 \%)$ y que en ambos sexos en población general (menor del $1 \%)$. Sólo unos pocos referían consumo de opiáceos y drogas de diseño. En conclusión, el patrón de consumo de sustancias en personas transexuales, excepto para la cocaína, es similar entre ambos sexos, y se asemeja al patrón de consumo masculino en población general. El consumo de cocaína es hasta diez veces mayor en el grupo de mujeres transexuales (H-M) con respecto a los otros grupos.

Palabras clave: Transexual; Transexualismo; Disforia de género; Patrones de consumo; Alcohol; Tabaco; Drogas.

\begin{abstract}
This study evaluated the patterns of substance use in a large sample of male-to-female (MtoF) and female-to-male (FtoM) transsexuals. A total of 251 transsexual subjects (163 MtoF and $88 \mathrm{FtoM}$ ), attended in the Catalonia Gender Unit, completed self-administrated questionnaires on consumption of alcohol, tobacco, cannabis, cocaine, opioids, and designer drugs. Results were compared with the general population in Catalonia using data from the National Health Service (EDADES 2013 study). Current consumption of alcohol (70.1\%), tobacco $(46.2 \%)$, and cannabis $(16.3 \%)$ among transsexuals was similar when compared with men $(72.1 \%, 42.1 \%, 12.8 \%)$ and increased when compared with women $(57.6 \%, 35.2 \%, 5 \%)$; the consumption between MtoF and FtoM subgroups was similar. The use of cocaine was almost ten times more prevalent in the MtoF subgroup than in the FtoM subgroup (1.1\%), and in general population (less than $1 \%$ ). Only a few reported uses of opioids and designer drugs. In conclusion, the substance use among transsexuals, except for the use of cocaine, was similar between MtoF and FtoM subgroups, and resembled the consumption prevalence among men in the general population. The proportion of cocaine consumers in the MtoF subgroup was up to ten times higher than in other subgroups.

Keywords: Transsexual; Transsexualism; Gender Dysphoria; Alcohol use; Consumption patterns; Alcohol; Tobacco; Drugs.
\end{abstract}

Recibido: Marzo 2017; Aceptado: Abril 2018.

Enviar correspondencia a:

Esther Gómez Gil. Institut Clínic de Neurociències. Servei de Psiquiatria. Hospital Clínic. Villarroel 170, 08036 Barcelona, Spain.

Tel: +34 93 (2275477). Fax: +34 (93) 2275477. E-mail: esgomez@clinic.ub.es / Isabel Esteva de Antonio. Departamento de Endocrinología.

Hospital Regional Universitario. Málaga. Mail: isabelestevadeantonio@gmail.com 
$\mathrm{E}$ 1 transexualismo (ICD-10, World Health Organization, 1993), trastorno de identidad de género (DSM-IV-TR, American Psychiatric Association, 2000), disforia de género (DSM-5, American Psychiatric Association, 2013), o incongruencia de género (Drescher, Cohen-Kettenis y Winter, 2012) es una condición en la que los individuos experimentan discrepancia entre el sexo asignado al nacer y el género con que se identifican, a menudo acompañado de gran malestar personal (The World Professional Association for Transgender Health (WPATH), 2011). La intensidad de la discrepancia es extrema cuando se utilizan los primeros dos términos (DSM-IV-TR, American Psychiatric Associasion, 2000; ICD10, World Health Organization, 1993) y más dimensional cuando se utiliza el término reciente de disforia de género (DSM-5, American Psychiatric Association, 2013).

A pesar del creciente interés científico por las personas con disforia de género, sus hábitos de consumo o abuso de alcohol, tabaco y drogas ilícitas aún carecen de una investigación relevante. En estudios realizados en las unidades de género especializadas, los datos de prevalencia de abuso de alcohol y/o de drogas varían según el género y el país: de $11,3 \%$ a $50 \%$ en personas transexuales hombre-a-mujer (H-M) y de $3,8 \%$ a $61,5 \%$ en personas transexuales mujer-a-hombre (M-H) (Cole, O’Boyle, Emory y Meyer, 1997; De Cuypere, Janes y Rubens, 1995; Gómez-Gil, Trilla, Salamero, Godás y Valdés, 2009; Haraldsen y Dahl, 2000; Hepp, Kraemer, Schnyder, Miller y Delsignore, 2005; Landen, Walinder y Lundstrom, 1998; Verschoor y Poortinga, 1988). Mientras que en los Países Bajos (Verschoor y Poortinga, 1988), Suiza (Hepp et al., 2005) y los Estados Unidos (Cole et al., 1997) la prevalencia fue superior entre transexuales H-M que en M-H, en Bélgica (De Cuypere et al., 1995) y en Suecia (Landen et al., 1998) fue mayor en el subgrupo M-H; sin embargo, ninguna de estas diferencias resultaron estadísticamente significativas. Un estudio español de nuestro equipo (Gómez-Gil et al., 2009) mostró que la prevalencia de abuso o dependencia en personas transexuales H-M era más alta que en el subgrupo M-H, tanto para el alcohol $(10,7 \%$ vs. $1,4 \%$ ) como para otras sustancias $(14,5 \%$ vs. $1,4 \%)$.

Los datos sobre el consumo de sustancias son aún más limitados que los datos sobre abuso en esta población. En los E.E.U.U., los datos sobre el consumo de sustancias se han obtenido de estudios en mujeres transgénero (H-M) sobre prevalencia y comportamientos de riesgo para infección por VIH. Una revisión sistemática (Herbst et al., 2008) demostró que la prevalencia de consumo de alcohol era del 43,7\%, de marihuana del 20,2\%, de drogas ilegales inyectadas del $12 \%$ y de otras drogas ilícitas del $26,7 \%$. En estudios posteriores, la proporción de consumidores fue similar (Reback y Fletcher, 2014; Santos et al., 2014; Sevelius, Reznick, Hart y Schwarcz, 2009) o más alta (Rowe, Santos, McFarland y Wilson, 2015). En dos de estos estudios (Reback et al., 2014; Santos et al., 2014), la prevalencia de consumo de alcohol y de drogas ilícitas en mujeres transexuales era más alta que en la población general. Un estudio canadiense reciente encontró mayor prevalencia de consumo de anfetaminas y cocaína entre las personas transexuales que en la población no transexual (Scheim, Bauer y Shokoohi, 2017). La mayoría de estos estudios, sin embargo, no incluyeron a los hombres transgénero (M-H) y además, como sus participantes fueron reclutadas principalmente en las calles, en agencias de servicios sociales específicas para personas transgénero, o a través de muestras dirigido por los propios encuestados, las comparaciones con estudios llevados a cabo en contextos clínicos deben hacerse con precaución.

Se sabe aún menos sobre los hábitos de fumar en personas con disforia de género. En dos estudios en población transgénero, los fumadores activos representaban un tercio (Conron, Scott, Stowell y Landers, 2012; Shires y Jaffee, 2016); también eran más fumadores que las personas no transgénero con un ratio de probabilidad del 2,7 (Conron et al., 2012).

Los objetivos de este estudio fueron: (1) evaluar los hábitos referidos de consumo de alcohol, tabaco, cannabis, cocaína, opiáceos y drogas de diseño en una amplia muestra de personas transexuales españolas H-M y M-H que acuden a la Unidad de Identidad de Género de Cataluña, España; y (2) comparar esta muestra con la población general.

\section{Método}

\section{Participantes}

Se ofreció a un total de 300 personas transexuales de forma consecutiva la posibilidad de participar en el estudio, durante una visita psiquiátrica en la Unidad de Identidad de Género del Hospital Clínico de Barcelona, desde el año 2010 a 2014. Se obtuvo el consentimiento informado de los participantes. El diagnóstico de transexualismo en la edad adulta o la adolescencia fue realizado por dos expertos en identidad de género, un psicólogo y un psiquiatra, usando los criterios de la CIE-10 (World Health Organization, 1993) o del DSM-IV-TR (American Psychiatric Associasion, 2000) . El estudio fue aprobado por el Comité de Ética del hospital y se llevó a cabo según la Declaración de Helsinki (World Medical Association, 2013).

Este estudio forma parte de un proyecto de investigación más extenso que ha evaluado la comorbilidad psiquiátrica (Gomez-Gil et al., 2009), el malestar social, la ansiedad y la depresión (Gomez-Gil et al., 2012), la personalidad (Gomez-Gil et al., 2013), la calidad de vida (Gomez-Gil, Zubiaurre-Elorza, de Antonio, Guillamon y Salamero, 2014) y la calidad de vida sexual (Bartolucci et al., 2015) de personas transexuales españolas.

\section{Mediciones}

Se trata de un estudio descriptivo transversal. Se realizó un cuestionario diseñado por los investigadores para 
recoger la información sobre la edad, el sexo y el estado de tratamiento hormonal de los participantes. Se utilizó una versión en español (Rubio Valladolid, Bermejo Vicedo, Caballero Sanchez-Serrano y Santo-Domingo Carrasco, 1998) del Alcohol Use Disorders Identification Test (AUDIT, Test de Identificación de Trastornos del Uso del Alcohol; (Saunders, Aasland, Babor, de la Fuente y Grant, 1993) para evaluar los hábitos de consumo y para detectar el posible consumo de riesgo y perjudicial de alcohol. Se empleó una puntuación de corte de 8 para el consumo de riesgo, con sensibilidad de $81,4 \%$ a $90 \%$ y especificidad de $90 \%$ a 94,6\% (de Torres et al., 2009; Gomez, Conde, Santana y Jorrin, 2005; Rubio Valladolid et al., 1998). Para evaluar los hábitos de fumar se utilizó una pregunta de respuesta múltiple: ¿Cuántos cigarrillos fumas por día? (No fumo; 1-15; 16-25; 26 ó más). Por último, un cuestionario diseñado por los investigadores evaluó el uso de otras sustancias adictivas con cuatro preguntas destinadas a evaluar el consumo actual de las siguientes drogas: (1) ¿Consumes cocaína? (Sí, No); (2) ¿Consumes cannabis o sus derivados? (Sí, No); (3) ¿Consumes drogas de diseño (éxtasis o éxtasis líquido)? (Sí, No); (4) ¿Consumes opiáceos (heroína, morfina, codeína, metadona, tramadol, fentanilo, buprex)? (Sí, No).

Se utilizó la prevalencia del consumo de alcohol, tabaco, cannabis, cocaína, éxtasis y heroína en los últimos 30 días en las mujeres, los hombres y en la muestra total tomada de la encuesta EDADES 2013 (datos recogidos entre 2013 y 2014) para comparar la muestra con la población general catalana (Generalitat de Catalunya Departament de Salut, 2015). EDADES es una encuesta que se administra en los hogares, y que evalúa el consumo de alcohol, tabaco, y drogas entre los residentes en España cada dos años. Un entrevistador entrenado pregunta a los participantes: "En los últimos 30 días ¿cuántos días ha consumido usted alcohol [fumado tabaco, consumido cannabis, consumido cocaína]?” y “¿Ha consumido éxtasis u otras drogas de diseño en los últimos 30 días?”. La muestra de Cataluña de EDADES 2013 incluyó a 2019 individuos hombres y mujeres de entre 15 y 64 años de edad y fue elegida debido a que el marco temporal de recogida es similar al de recopilación de los datos en la muestra de población transexual.

\section{Análisis estadístico}

Los datos de nuestra muestra se analizaron utilizando el Paquete Estadístico para Ciencias Sociales (SPSS)-versión 18. Se calcularon las frecuencias, medias y desviaciones típicas para describir la muestra. Se verificaron los datos de normalidad de la distribución y los valores atípicos. Los valores $p$ por debajo de 0,05 se consideraron estadísticamente significativos. Para comparar las variables cuantitativas, se utilizó la prueba $t$ de Student bilateral. Se utilizó la prueba de chi cuadrado para comparar la prevalencia entre los participantes H-M y M-H. La prueba exacta de Fisher se utilizó cuando era necesario. Cuando se encontraron diferencias estadísticamente significativas con la prueba $\mathrm{t}$ de Student se empleó el análisis multivariado de regresión logística para controlar los efectos de la edad, el sexo y el tratamiento hormonal. Para comparar la prevalencia de alcohol y consumo de drogas ilícitas entre nuestra muestra y la muestra de la población general catalana se utilizó la prueba de $\mathrm{Z}$ de una muestra de dos colas para una proporción de la población (disponible en línea en http:// epitools.ausvet.com.au/content.php?page=z-test-1).

\section{Resultados}

\section{Participantes}

De las 300 personas transexuales que fueron invitados a participar, $22(7,3 \%)$ no accedieron a participar y $27(9 \%)$ fueron excluidas debido a respuestas incompletas en los cuestionarios. Se incluyó finalmente un total de $251(83,7 \%)$ sujetos (163 H-M y $88 \mathrm{M}-\mathrm{H})$ (rango de edad 14 - 63 años, edad media $=29,9 ; S D=10,26)$. El subgrupo H-M era mayor que el subgrupo M-H (rango de edad 14- 63 años, edad media $=31,47 ; S D=10,91$ vs. rango de edad 18-51 años, edad media $=26,99 ; S D=8,23 ; t(222,76)=3,656, p<0,05)$. De todos los participantes, $111(44,2 \%)$ estaban en tratamiento hormonal en el momento de participación en el estudio. La diferencia entre la proporción de personas en tratamiento hormonal entre el subgrupo H-M $(n=87 ; 53,4 \%)$ y el subgrupo M-H $(n=24 ; 27,3 \%)$ fue estadísticamente significativa $(z=2,035, p=0,042)$. Otras características socio-demográficas han sido descritas en estudios realizados con submuestras de este estudio; más del $50 \%$ de la muestra tenía bajo nivel educativo y un empleo de baja cualificación (Gómez-Gil et al., 2013; Gómez-Gil et al., 2012).

\section{Patrones de uso de sustancias y diferencias entre los subgrupos $\boldsymbol{H}-\mathbf{M}$ y $\mathbf{M}-\mathrm{H}$}

La única diferencia estadísticamente significativa entre los subgrupos H-M y M-H fue en el consumo de cocaína: el porcentaje de consumidores de cocaína en el subgrupo H-M fue casi diez veces mayor (Tabla 1). No hubo diferencias estadísticamente significativas entre los subgrupos H-M y M-H (Tabla 2) en la proporción de bebedores de riesgo o consumo perjudicial ni en la puntuación media de la escala AUDIT ( $M=2,6 ; S D=3,33$ vs. $M=2,52 ; S D=3,52)$. Los resultados de los análisis de regresión logística multivariado ajustados por edad, sexo y tratamiento hormonal revelaron una asociación entre el consumo de cocaína y el subgrupo H-M $(O R=7,8 ; p=0,05)$. El modelo explicó el $10,5 \%$ de la varianza según la $R^{2}$ de Nagelkerke.

\section{Diferencias en el uso de sustancias entre sujetos transexuales en comparación con la población general catalana}

La Tabla 1 muestra la prevalencia del consumo de alcohol, tabaco, cannabis, cocaína, opiáceos y drogas de diseño 
Tabla 1. Prevalencia del consumo de sustancias entre transexuales varón-mujer (H-M) y mujer-varón (M-H) y en la población general de Cataluña de la encuesta EDADES 2013 (Generalitat de Catalunya Departament de Salut, 2015).

\begin{tabular}{|c|c|c|c|c|c|c|}
\hline & $\begin{array}{c}\text { Total } \\
\text { Transexuales } \\
(n=\mathbf{2 5 1})\end{array}$ & $\begin{array}{c}H-M \\
(n=163)\end{array}$ & $\begin{array}{c}M-H \\
(n=88)\end{array}$ & $\begin{array}{c}\text { Total Población } \\
\text { General } \\
(n=2019)\end{array}$ & $\begin{array}{l}\text { Hombres } \\
(n=1027)\end{array}$ & $\begin{array}{c}\text { Mujeres } \\
(n=992)\end{array}$ \\
\hline & N (\%) & n (\%) & n (\%) & N (\%) & n (\%) & n (\%) \\
\hline Consumidores de alcohol (puntuación AUDIT) & $176(70,1 \%)$ & $114(69,9 \%)$ & $62(70,5 \%)$ & $65,0 \%$ & $72,1 \%$ & $57,6 \%$ \\
\hline Bebedores de bajo riesgo (1-7) & $159(63,3 \%)$ & $103(63,2 \%)$ & $56(63,6 \%)$ & & & \\
\hline Bebedores de alto riesgo (8-19) & $15(6 \%)$ & $10(6,1 \%)$ & $5(5,6 \%)$ & & & \\
\hline Probable dependencia del alcohol $(\geq 20)$ & $2(0,8 \%)$ & $1(0,6 \%)$ & $1(1,1 \%)$ & & & \\
\hline Bebedores de riesgo o perjudicial $(\geq 8)$ & $17(6,8 \%)$ & $11(6,7 \%)$ & $6(6,8 \%)$ & & & \\
\hline Consumidores de tabaco & $116(46,2 \%)$ & $71(43,6 \%)$ & $45(51,1 \%)$ & $38,7 \%$ & $42,1 \%$ & $35,2 \%$ \\
\hline 1-15 por día & $89(76,7 \%)$ & $52(73,2 \%)$ & $37(82,2 \%)$ & & & \\
\hline 16-25 por día & $22(19 \%)$ & $16(22,5 \%)$ & $6(13,3 \%)$ & & & \\
\hline 26 o más por día & $5(4,3 \%)$ & $3(4,2 \%)$ & $2(4,4 \%)$ & & & \\
\hline Consumidores de cocaína & $17(6,8 \%)$ & $16(9,8 \%)$ & $1(1,1 \%)$ & $1,6 \%$ & $2,5 \%$ & $0,7 \%$ \\
\hline Consumidores de opiáceos & $1(0,4 \%)$ & o (o\%) & $1(1,1 \%)$ & * & * & * \\
\hline Consumidores de drogas de diseño & $4(1,6 \%)$ & $4(2,5 \%)$ & o (o\%) & $0,1 \%$ & $0,2 \%$ & $0,1 \%$ \\
\hline
\end{tabular}

Nota. * No hay datos disponibles para una comparación.

Tabla 2. Comparación del consumo de sustancias en transexuales varón-mujer (H-M) y mujer -varón (M-H) y en la población de hombres y mujeres de Cataluña de la encuesta EDADES 2013 (Generalitat de Catalunya Departament de Salut, 2015).

\begin{tabular}{|c|c|c|c|c|c|c|c|c|c|c|c|c|}
\hline & \multicolumn{2}{|c|}{ H-M vs. M-H } & \multicolumn{2}{|c|}{$\begin{array}{c}\text { Total de transexuales } \\
\text { vs. Total Población } \\
\text { General }\end{array}$} & \multicolumn{2}{|c|}{ H-M vs. Hombres } & \multicolumn{2}{|c|}{ H-M vs. Mujeres } & \multicolumn{2}{|c|}{ M-H vs. Hombres } & \multicolumn{2}{|c|}{ M-H vs. Mujeres } \\
\hline & $x^{2}$ & $p$ & $z$ & $p$ & $z$ & $p$ & $z$ & $p$ & $z$ & $p$ & $z$ & $p$ \\
\hline $\begin{array}{l}\text { Consumidores } \\
\text { de alcohol }\end{array}$ & 0,007 & n.s. & 1,7 & $\mathrm{n}, \mathrm{s}$, & 0,6 & n.s & 3,2 & 0,0015 & 0,3 & n.s. & 2,4 & 0,0143 \\
\hline $\begin{array}{l}\text { Consumidores } \\
\text { de tabaco }\end{array}$ & 1,32 & n.s. & 2,4 & 0,0147 & 0,4 & n.s. & 2,2 & 0,0247 & 1,7 & n.s. & 3,1 & 0,0018 \\
\hline $\begin{array}{l}\text { Consumidores } \\
\text { de cannabis }\end{array}$ & 0,883 & n.s. & 4,1 & $<0,0001$ & 0,7 & n.s. & 5,7 & $<0,0001$ & 1,8 & n.s. & & * \\
\hline $\begin{array}{l}\text { Consumidores } \\
\text { de cocaína }\end{array}$ & 6,818 & 0,009 & & & & & & $\approx$ & & & & * \\
\hline $\begin{array}{l}\text { Consumidores } \\
\text { de opiáceos }\end{array}$ & $0,351^{\star}$ & $\mathrm{n}, \mathrm{s}$, & & & & & & & & & & \\
\hline $\begin{array}{l}\text { Consumidores de } \\
\text { drogas de diseño }\end{array}$ & $0,301^{*}$ & $\mathrm{n}, \mathrm{s}$, & & & & & & $*$ & & & & $*$ \\
\hline
\end{tabular}

Nota. * Se utilizó la prueba exacta de Fisher; ** Comparación no fiable debido al escaso tamaño muestral; n.s.: no estadísticamente significativa.

nuestra muestra de personas transexuales y en la de población general tomada de la encuesta EDADES de 2013. Como se observa en la Tabla 2, las muestras totales no mostraron diferencias estadísticamente significativas en el consumo de alcohol, pero el porcentaje de consumidores de tabaco y cannabis fue mayor en los sujetos transexuales. Sin embargo, en las comparaciones entre los subgrupos, la prevalencia del uso de alcohol, tabaco y cannabis, tanto en las personas transexuales H-M como en el subgrupo M-H, no difirió en comparación con los hombres y sólo se incrementó en comparación con las mujeres no transexuales.

A pesar de que una comparación estadística no fue posible, el consumo de la cocaína en el subgrupo H-M (9,8\%) fue notablemente mayor que en los M-H (9 veces supe- rior), y que en los hombres (casi 4 veces mayor) y las mujeres (14 veces mayor) (Tabla 1$)$.

\section{Discusión}

Según nuestro conocimiento, este estudio es el primero en examinar los patrones de consumo de sustancias en una amplia muestra de personas transexuales de ambos sexos en Cataluña y comparar su prevalencia de consumo con la de la población general. La evaluación de estos hábitos entre las personas transexuales es importante a la hora de planificar políticas de cuidados preventivos, ya que el abuso de sustancias se asocia con otros factores de riesgo, como el elevado riesgo de exclusión social (Hyde et al., 2014; 
Scheim et al., 2017), la implicación en trabajo sexual (Sausa, Keatley y Operario, 2007) y la infección por VIH (Reback et al., 2014; Sausa et al., 2007), especialmente entre las mujeres transexuales $(\mathrm{H}-\mathrm{M})$.

La proporción de personas transexuales que consumen alcohol, tabaco, cannabis, opiáceos y drogas de diseño fue sorprendentemente similar entre los subgrupos H-M y los $\mathrm{M}-\mathrm{H}$; parece como si la disforia de género redujera las diferencias de género en el consumo observado a menudo en la población general, en la que los hombres tienden a consumir más sustancias que las mujeres. La única diferencia estadísticamente significativa fue en el consumo de cocaína y era considerablemente mayor en el subgrupo H-M. Las razones plausibles de esta diferencia en el consumo de cocaína puede ser, más que el sexo asignado al nacer o la identificación con el otro género, la frecuente participación en trabajos sexuales de las mujeres transexuales (H-M) en España. En un estudio previo de nuestro equipo (Gomez-Gil et al., 2009), el 33\% de las personas transexuales H-M informó de su participación anterior o actual en la prostitución y en espectáculos de sexo. El consumo de drogas puede mitigar los efectos negativos emocionales del trabajo sexual y podría ser requerido por los clientes de estas mujeres (Sausa et al., 2007). Por otro lado, estas sustancias ilícitas pueden ser utilizadas para aumentar la excitación sexual o disminuir el umbral del dolor (Dolengevich-Segal, Rodriguez-Salgado, Bellesteros-Lopez y Molina-Prado, 2017).

En comparación con los escasos datos de consumo existentes en la literatura, en estudios de los Estados Unidos (Herbst et al., 2008; Reback et al., 2014; Santos et al., 2014; Sevelius et al., 2009) las mujeres transexuales refieren un menor consumo de alcohol, pero un mayor consumo de cannabis que las mujeres transexuales en nuestro estudio. Es imposible comparar el consumo de otras sustancias debido a las distintas categorías utilizadas. La prevalencia de tabaquismo (Conron et al., 2012; Shires et al., 2016) fue menor que en nuestro estudio ( $36,2 \%$ y $27,2 \%$ vs. $46,2 \%)$. Sin embargo, debe tenerse en cuenta que la prevalencia de fumadores $(17,3 \%)$ en población USA fue también menor (Conron et al., 2012) que en la población general catalana $(38,7 \%)$.

Curiosamente, al comparar por subgrupos, la proporción de consumidores de alcohol, tabaco y cannabis en las personas transexuales $\mathrm{H}-\mathrm{M}$ y $\mathrm{M}-\mathrm{H}$ se asemejó a la prevalencia en el grupo de hombres y era más alta que en el grupo de mujeres no transexuales. A pesar de que no fue posible una comparación estadística, la prevalencia del uso de cocaína en el subgrupo H-M era notablemente mayor en comparación con los hombres y las mujeres de la población general catalana. Este aumento podría estar relacionado, como ya se ha mencionado arriba, con el ambiente marginal y socioeconómicamente peor de algunas mujeres transexuales involucradas en el comercio sexual. Se necesitan más investigaciones para determinar las causas del patrón de consumo de alcohol, tabaco y cannabis de personas transexuales más similar al masculino de la población general.

La presente investigación tiene varias limitaciones. En primer lugar, los participantes fueron reclutados a través de una Unidad de Género cuando solicitaban tratamiento. Debido al temor a consecuencias negativas en la asistencia clínica por el consumo, la prevalencia referida de éste hábito puede ser inferior a la real. En segundo lugar, se estimó el consumo actual de sustancias sin tener en cuenta la prevalencia de uso/abuso a lo largo de la vida, ni criterios de dependencia, posiblemente, reduciendo la magnitud del problema. Por otra parte, la mayoría de las preguntas empleadas para evaluar el consumo de drogas no consideran la cantidad y la frecuencia de consumo. En tercer lugar, se utilizó un grupo control de otro estudio para las comparaciones (EDADES 2013) y los archivos de los datos originales del grupo de control no estaban disponibles. Finalmente, puesto que los participantes asistían a servicios médicos especializados, no está claro si estos resultados pueden aplicarse a quienes no solicitan atención en unidades clínicas. Nuestra hipótesis es que las personas transexuales que nunca han recibido atención médica o las que están tomando hormonas sin prescripción o control médico pueden tener peor calidad de vida que los asistentes a servicios especializados. Por lo tanto, el consumo de alcohol, tabaco y drogas ilícitas detectado en nuestro estudio puede ser subestimado con respecto al total de la población transexual.

En conclusión, el uso de sustancias entre las personas transexuales fue similar entre los subgrupos $\mathrm{H}-\mathrm{M}$ y $\mathrm{M}-\mathrm{H}$, excepto el uso de la cocaína. En comparación con la población general catalana, las personas transexuales se asemejaron al grupo de los hombres en el uso de alcohol, tabaco y cannabis, pero la prevalencia fue mayor que la del grupo de mujeres. La prevalencia de consumo de cocaína en las mujeres transexuales fue mayor en comparación con los hombres transexuales y con los hombres y las mujeres de la población general. Sólo unos pocos individuos informaron del uso de opiáceos y drogas de diseño. Se necesita más investigación para examinar las relaciones causales de los resultados. Teniendo en cuenta los riesgos asociados al uso de sustancias, cualquier intervención dirigida a mejorar la salud de las personas transexuales debe considerar los patrones particulares de consumo de alcohol, tabaco y drogas ilegales.

\section{Agradecimientos}

Este estudio fue subvencionado por el Ministerio de Igualdad Español (MI), Instituto de la Mujer, subvención IMG2009-PI040964 (EG-G, IE y MS), y el Ministerio de Economía e Innovación, subvención PSI2014-58004-P (AG y EG-G). 


\section{Conflicto de intereses}

No hay ningún conflicto de interés.

\section{Referencias}

American Psychiatric Association. (2000). Diagnostic and statistical manual of mental disorders: DSM-IV-TR. 4th ed. Washington, DC: APA.

American Psychiatric Association. (2013). Diagnostic and statistical manual of mental disorders: DSM-5. 5 th ed. Washington, DC: APA.

Bartolucci, C., Gómez-Gil, E., Salamero, M., Esteva, I., Guillamón, A., Zubiaurre, L., ... Montejo, A. L. (2015). Sexual quality of life in gender-dysphoric adults before genital sex reassignment surgery. The Journal of Sexual Medicine, 12, 180-188. doi:10.1111/jsm.12758.

Cole, C. M., O’Boyle, M., Emory, L. E. y Meyer, W. J., 3rd. (1997). Comorbidity of gender dysphoria and other major psychiatric diagnoses. Archives of Sexual Behavior, 26, 13-26.

Conron, K. J., Scott, G., Stowell, G. S. y Landers, S. J. (2012). Transgender health in Massachusetts: results from a household probability sample of adults. American Journal of Public Health, 102, 118-122. doi:10.2105/ AJPH.2011.300315.

De Cuypere, G., Janes, C. y Rubens, R. (1995). Psychosocial functioning of transsexuals in Belgium. Acta Psychiatrica Scandinavica, 91, 180-184.

de Torres, L. A., Rebollo, E. M., Ruiz-Moral, R., Fernández-García, J. A., Vega, R. A. y Palomino, M. M. (2009). Diagnostic usefulness of the Alcohol Use Disorders Identification Test (AUDIT) questionnaire for the detection of hazardous drinking and dependence on alcohol among Spanish patients. European Journal of General Practice, 15, 15-21. doi:10.1080/13814780902855754.

Dolengevich-Segal, H., Rodríguez-Salgado, B., Bellesteros-López, J. y Molina-Prado, R. (2017). Chemsex. An emergent phenomenon. Adicciones, 29, 207-209. doi:10.20882/adicciones.894.

Drescher, J., Cohen-Kettenis, P. y Winter, S. (2012). Minding the body: situating gender identity diagnoses in the ICD-11. International Review of Psychiatry, 24, 568-577. do i:10.3109/09540261.2012.741575.

Generalitat de Catalunya Departament de Salut. (2015). Informe dels resultats per a Catalunya de l'Enquesta domiciliària sobre alcohol $i$ drogues a Espanya (EDADES) 2013. Recuperado de http://drogues.gencat.cat/ca/professionals/epidemiologia/estudes/.

Gómez-Gil, E., Gutierrez, F., Cañizares, S., Zubiaurre-Elorza, L., Monràs, M., Esteva de Antonio, I., ... Guillamón, A. (2013). Temperament and character in transsexuals. Psychiatry Research, 210, 969-974. doi:10.1016/j.psychres.2013.07.040.
Gómez-Gil, E., Trilla, A., Salamero, M., Godás, T. y Valdés, M. (2009). Sociodemographic, clinical, and psychiatric characteristics of transsexuals from Spain. Archives of Sexual Behavior, 38, 378-392. doi:10.1007/s10508-007-9307-8.

Gómez-Gil, E., Zubiaurre-Elorza, L., Esteva de Antonio, I., Guillamón, A. y Salamero, M. (2014). Determinants of quality of life in Spanish transsexuals attending a gender unit before genital sex reassignment surgery. Quality of Life Research, 23, 669-676. doi:10.1007/s11136-013-0497-3.

Gómez-Gil, E., Zubiaurre-Elorza, L., Esteva, I., Guillamón, A., Godás, T., Almaraz, M. C., ... Salamero, M. (2012). Hormone-treated transsexuals report less social distress, anxiety and depression. Psychoneuroendocrinology, 37, 662-670. doi:10.1016/j.psyneuen.2011.08.010.

Gómez, A., Conde, A., Santana, J. M. y Jorrín, A. (2005). Diagnostic usefulness of brief versions of Alcohol Use Disorders Identification Test (AUDIT) for detecting hazardous drinkers in primary care settings. Journal of Studies on Alcohol and Drugs, 66, 305-308.

Haraldsen, I. R. y Dahl, A. A. (2000). Symptom profiles of gender dysphoric patients of transsexual type compared to patients with personality disorders and healthy adults. Acta Psychiatrica Scandinavica, 102, 276-281.

Hepp, U., Kraemer, B., Schnyder, U., Miller, N. y Delsignore, A. (2005). Psychiatric comorbidity in gender identity disorder. Journal of Psychosomatic Research, 58, 259-261. doi:10.1016/j.jpsychores.2004.08.010.

Herbst, J. H., Jacobs, E. D., Finlayson, T. J., McKleroy, V. S., Neumann, M. S., Crepaz, N. y Hiv Aids Prevention Research Synthesis Team. (2008). Estimating HIV prevalence and risk behaviors of transgender persons in the United States: a systematic review. AIDS and Behavior, 12, 1-17. doi:10.1007/s10461-007-9299-3.

Hyde, Z., Doherty, M., Tilley, P. J. M., McCaul, K. A., Rooney, R. y Jancey, J. (2014). The First Australian National Trans Mental Health Study: Summary of Results. Recuperado de https:/ /www.beyondblue.org.au/docs/default-source/ research-project-files/bw0288_the-first-australian-national-trans-mental-health-study-summary-of-results.pdf?sfvrsn=2.

Landen, M., Walinder, J. y Lundstrom, B. (1998). Clinical characteristics of a total cohort of female and male applicants for sex reassignment: a descriptive study. Acta Psychiatrica Scandinavica, 97, 189-194.

Reback, C. J. y Fletcher, J. B. (2014). HIV prevalence, substance use, and sexual risk behaviors among transgender women recruited through outreach. AIDS and Behavior, 18, 1359-1367. doi:10.1007/s10461-013-0657-z.

Rowe, C., Santos, G. M., McFarland, W. y Wilson, E. C. (2015). Prevalence and correlates of substance use among trans female youth ages 16-24 years in the San Francisco Bay Area. Drug Alcohol Depend, 147, 160-166. doi:10.1016/j.drugalcdep.2014.11.023. 
Rubio Valladolid, G., Bermejo Vicedo, J., Caballero Sánchez-Serrano, M. C. y Santo-Domingo Carrasco, J. (1998). Validation of the Alcohol Use Disorders Identification Test (AUDIT) in primary care. Revista Clinica Española, 198, 11-14.

Santos, G. M., Rapues, J., Wilson, E. C., Macias, O., Packer, T., Colfax, G. y Raymond, H. F. (2014). Alcohol and substance use among transgender women in San Francisco: prevalence and association with human immunodeficiency virus infection. Drug and Alcohol Review, 33, 287-295. doi:10.1111/dar.12116.

Saunders, J. B., Aasland, O. G., Babor, T. F., de la Fuente, J. R. y Grant, M. (1993). Development of the Alcohol Use Disorders Identification Test (AUDIT): WHO Collaborative project on early detection of persons with harmful alcohol consumption-II. Addiction, 88, 791-804.

Sausa, L. A., Keatley, J. y Operario, D. (2007). Perceived risks and benefits of sex work among transgender women of color in San Francisco. Archives of Sexual Behavior, 36, 768-777. doi:10.1007/s10508-007-9210-3.

Scheim, A. I., Bauer, G. R. y Shokoohi, M. (2017). Drug use among transgender people in Ontario, Canada: Disparities and associations with social exclusion. Addictive Behaviors, 72, 151-158. doi:10.1016/j.addbeh.2017.03.022.

Sevelius, J. M., Reznick, O. G., Hart, S. L. y Schwarcz, S. (2009). Informing interventions: the importance of contextual factors in the prediction of sexual risk behaviors among transgender women. AIDS Education and Prevention, 21, 113-127. doi:10.1521/aeap.2009.21.2.113.

Shires, D. A. y Jaffee, K. D. (2016). Structural discrimination is associated with smoking status among a national sample of transgender individuals. Nicotine y Tobacco Research, 18, 1502-1508. doi:10.1093/ntr/ntv221.

The World Professional Association for Transgender Health (WPATH). (2011). Standarts of Care for the Health of Transsexual, Transgender and Gender Nonconforming People V7. Atlanta. Recuperado de http:/ /www.wpath.org/ site_page.cfm?pk_association_webpage_menu=1351.

Verschoor, A. M. y Poortinga, J. (1988). Psychosocial differences between Dutch male and female transsexuals. Archives of Sexual Behavior, 17, 173-178.

World Health Organization. (1993). The ICD-10 classification of mental and behavioural disorders: Diagnostic criteria for research. Geneva: WHO.

World Medical Association. (2013). World Medical Association Declaration of Helsinki: ethical principles for medical research involving human subjects. JAMA, 310, 2191-2194. doi:10.1001/jama.2013.281053. 